\title{
FIRST PASSAGE OF A MARKOV ADDITIVE PROCESS AND GENERALIZED JORDAN CHAINS
}

\author{
BERNARDO D'AURIA, * Universidad Carlos III de Madrid \\ JEVGENIJS IVANOVS, ${ }^{* *}$ Eurandom and University of Amsterdam \\ OFFER KELLA, ${ }^{* * *}$ The Hebrew University of Jerusalem \\ MICHEL MANDJES, ${ }^{* * * *}$ University of Amsterdam, Eurandom and CWI
}

\begin{abstract}
In this paper we consider the first passage process of a spectrally negative Markov additive process (MAP). The law of this process is uniquely characterized by a certain matrix function, which plays a crucial role in fluctuation theory. We show how to identify this matrix using the theory of Jordan chains associated with analytic matrix functions. This result provides us with a technique that can be used to derive various further identities.
\end{abstract}

Keywords: Lévy process; fluctuation theory; Markov additive process

2010 Mathematics Subject Classification: Primary 60K25

Secondary $60 \mathrm{~K} 37$

\section{Introduction}

Continuous-time Markov additive processes (MAPs) with one-sided jumps have proven to be an important modelling tool in various application areas, such as communications networking [19, Chapters 6-7] and finance [5], [14]. Over the past decades a vast body of literature has been developed; see, for instance, [2, Chapter XI] for a collection of results. A MAP can be thought of as a Lévy process whose Laplace exponent depends on the state of a (finite-state) Markovian background process (with additional jumps at transition epochs of this background process). It is a nontrivial generalization of the standard Lévy process, with many analogous properties and characteristics, as well as new mathematical objects associated to it, posing new challenges. Any Lévy process is characterized by a Laplace exponent $\psi(\alpha)$; its counterpart for MAPs is the matrix exponent $F(\alpha)$, which is essentially a multidimensional analogue of $\psi(\alpha)$.

In this paper we consider the first passage process $\tau_{x}$ defined as the first time the process exceeds level $x$. We concentrate on the case of a spectrally negative MAP (that is, all jumps are negative), so that the first passage process is a MAP itself. Knowledge of the matrix exponent of this process, which in the sequel we denote by the matrix function $\Lambda(q)$, is of crucial interest when addressing related fluctuation theory issues. Indeed, it can be considered as the multidimensional generalization of $-\Phi(q)$, where $\Phi(q)$ is the (one-dimensional) right inverse of $\psi(\alpha)$, as given in [15, Equation (3.15)]. Our main result concerns the identification

\footnotetext{
Received 5 July 2010; revision received 15 September 2010.

* Postal address: Universidad Carlos III de Madrid, Avda Universidad 30, 28911 Leganes (Madrid), Spain.

** Postal address: Eurandom, PO Box 513, 5600 MB Eindhoven, The Netherlands.

Email address: ivanovs@eurandom.tue.nl

*** Postal address: Department of Statistics, The Hebrew University of Jerusalem, Jerusalem 91905, Israel.

**** Postal address: Korteweg-de Vries Institute for Mathematics, University of Amsterdam, Science Park 904, 1098 XH Amsterdam, The Netherlands.
} 
of the matrix function $\Lambda(q)$ in terms of the matrix exponent $F(\alpha)$ of the original MAP. We provide the Jordan normal form of $\Lambda(q)$, relying on the theory of Jordan chains associated with analytic matrix functions.

The problem of identification of $\Lambda(q)$ received a lot of attention in the literature. It is known that $\Lambda(q)$ is a unique (admissible) solution of a certain matrix integral equation. This result in different degrees of generality appears in [1], [6], [8], [17], [18], [20], and [21]. Alternatively, we can use an iterative method to compute $\Lambda(q)$; see, for example, [6]. Some spectral considerations (under the assumption that $\Lambda(q)$ has distinct eigenvalues) can be found in [1], [6], and [20]. It is plausible that an iterative method is preferable if the aim is to compute $\Lambda(q)$ numerically. Our result, however, provides a better understanding of how $\Lambda(q)$ is related to $F(\alpha)$, and can be used to prove various further identities. As an example, we provide a simple proof of the fact that $\Lambda(q)$ is the unique solution of the abovementioned integral equation.

It has been realized before that the 0 s of $\operatorname{det}(F(\alpha))$ with positive real parts and the corresponding null spaces of $F(\alpha)$ play an important role in many problems concerning fluctuations of MAPs; see, for example, [3], [4], and [13]. The problem is that we have to assume that $\operatorname{det}(F(\alpha))$ has a sufficient number of distinct 0s. The number of 0s was determined in [13], and in [12] it was shown that if a MAP is time reversible then the 0s are semi-simple, that is, they can be treated as distinct. In general though, this is not the case. In this paper we provide a final answer to the above problem through the use of generalized Jordan chains associated to $F(\alpha)$. A number of examples are given in the extended version of this paper [7].

This paper is organized as follows. In Section 2 we review some main results from analytic matrix function theory, while in Section 3 we identify the matrix exponent $\Lambda(q)$ by relating the Jordan pairs of the matrix functions $F(\alpha)-q \mathbb{I}$ and $\alpha \mathbb{I}+\Lambda(q)$ for a fixed $q \geq 0$, with $\mathbb{I}$ being the identity matrix,. This result, which is Theorem 1 and which can be considered as the main contribution of our work, is explicit in the sense that it is given in terms of computable quantities associated with $F(\alpha)$. Finally, in Section 4 we discuss applicability of our results.

The remainder of this section is devoted to the definitions of the quantities of interest, with a focus on spectrally negative MAPs and their first passage process. Throughout this work, we use bold symbols to denote column vectors unless otherwise specified. In particular, $\mathbf{1}$ and $\mathbf{0}$ are the vectors of $1 \mathrm{~s}$ and $0 \mathrm{~s}$, respectively, and $\boldsymbol{e}_{i}$ is a vector with a 1 in the $i$ th element and $0 \mathrm{~s}$ elsewhere.

\subsection{Spectrally negative MAP}

A MAP is a bivariate Markov process $(X(t), J(t))$ defined as follows. Let $J(\cdot)$ be an irreducible continuous-time Markov chain with finite state space $E=\{1, \ldots, N\}$, transition rate matrix $Q=\left(q_{i j}\right)$, and a (unique) stationary distribution $\pi$. For each state $i$ of $J(\cdot)$, let $X_{i}(\cdot)$ be a Lévy process with Laplace exponent $\psi_{i}(\alpha)=\log \left(\mathrm{E}^{\alpha X_{i}(1)}\right)$. Letting $T_{n}$ and $T_{n+1}$ be two successive transition epochs of $J(\cdot)$, and given that $J(\cdot)$ jumps from state $i$ to state $j$ at $T_{n}$, we define the additive process $X(\cdot)$ in the time interval $\left[T_{n}, T_{n+1}\right)$ through

$$
X(t)=X\left(T_{n}-\right)+U_{i j}^{n}+\left[X_{j}(t)-X_{j}\left(T_{n}\right)\right]
$$

where $\left(U_{i j}^{n}\right)$ is a sequence of independent and identically distributed random variables with moment generating function

$$
\tilde{G}_{i j}(\alpha)=\mathrm{Ee}^{\alpha U_{i j}^{1}}, \quad \text { where } \quad U_{i i}^{1} \equiv 0,
$$

describing the jumps at transition epochs. To make the MAP spectrally negative, it is required that $U_{i j}^{1} \leq 0$ (for all $i, j \in\{1, \ldots, N\}$ ) and that $X_{i}(\cdot)$ is allowed to have only negative jumps 
(for all $i \in\{1, \ldots, N\}$ ). As a consequence, the moment generation functions $\tilde{G}_{i j}(\alpha)$ are well defined for $\alpha \geq 0$.

A Lévy process is called a downward subordinator if it has nonincreasing paths almost surely (a.s.). We denote the subset of indices of $E$ corresponding to such processes by $E_{\downarrow}$. Also, let $E_{+}=E \backslash E_{\downarrow}, N_{\downarrow}=\left|E_{\downarrow}\right|$, and $N_{+}=\left|E_{+}\right|$. It is convenient to assume that $E_{+}=\left\{1, \ldots, N_{+}\right\}$, which we do throughout this work. We use $\boldsymbol{v}_{+}$and $\boldsymbol{v}_{\downarrow}$ to denote the restrictions of a vector $\boldsymbol{v}$ to the indices from $E_{+}$and $E_{\downarrow}$, respectively. Finally, in order to exclude trivialities, it is assumed that $N_{+}>0$.

Define the matrix $F(\alpha)$ through

$$
F(\alpha)=Q \circ \tilde{G}(\alpha)+\operatorname{diag}\left[\psi_{1}(\alpha), \ldots, \psi_{N}(\alpha)\right],
$$

where $\tilde{G}(\alpha)=\left(\tilde{G}_{i j}(\alpha)\right)$; for matrices $A$ and $B$ of the same dimensions, we define $A \circ B=$ $\left(a_{i j} b_{i j}\right)$. We can see that in the absence of positive jumps $F(\alpha)$ is analytic on $\mathbb{C}^{\mathrm{Re}>0}=\{\alpha \in$ $\mathbb{C}: \operatorname{Re}(\alpha)>0\}$. Moreover, it is known that

$$
\mathrm{E}_{i}\left[\mathrm{e}^{\alpha X(t)} ; J(t)=j\right]:=\mathrm{E}_{i}\left[\mathrm{e}^{\alpha X(t)} 1_{\{J(t)=j\}}\right]=\left(\mathrm{e}^{F(\alpha) t}\right)_{i j}
$$

(cf. [2, Proposition XI.2.2]), where $\mathrm{E}_{i}(\cdot)$ denotes expectation given that $J(0)=i$ and $1_{\{\cdot\}}$ denotes the indicator function. We also write $\mathrm{E}\left[\mathrm{e}^{\alpha X(t)} ; J(t)\right]$ to denote the matrix with $i j$ th element given in (1). Hence, $F(\alpha)$ can be seen as the multidimensional analog of a Laplace exponent, defining the law of the MAP. In the following we call $F(\alpha)$ the matrix exponent of the MAP $(X(t), J(t))$.

An important quantity associated to a MAP is the asymptotic drift:

$$
\kappa=\lim _{t \rightarrow \infty} \frac{1}{t} X(t)=\sum_{i} \pi_{i}\left(\psi_{i}^{\prime}(0)+\sum_{j \neq i} q_{i j} \tilde{G}_{i j}^{\prime}(0)\right),
$$

which does not depend on the initial state $i$ of $J(t)$ [2, Corollary XI.2.7]. Finally, for $q \geq 0$, we define $F^{q}(\alpha)=F(\alpha)-q \mathbb{I}$, which can be seen as the matrix exponent of the MAP 'killed' at random time $e_{q}$ :

$$
\mathrm{E}\left[\mathrm{e}^{\alpha X(t)} ; t<e_{q}, J(t)\right]=\mathrm{e}^{(F(\alpha)-q \mathbb{I}) t},
$$

where $e_{q}$ is an exponential random variable of rate $q$ independent of everything else and $e_{0} \equiv \infty$ by convention.

\subsection{First passage process}

Define the first passage time over level $x>0$ for the (possibly killed) process $X(t)$ as

$$
\tau_{x}=\inf \{t \geq 0: X(t)>x\} .
$$

It is known that on $\left\{J\left(\tau_{x}\right)=i\right\}$ the process $\left(X\left(t+\tau_{x}\right)-X\left(\tau_{x}\right), J\left(t+\tau_{x}\right)\right), t \geq 0$, is independent from $(X(t), J(t)), t \in\left[0, \tau_{x}\right]$, and has the same law as the original process under $\mathrm{P}_{i}$. Therefore, in the absence of positive jumps the time-changed process $J\left(\tau_{x}\right)$ is a time-homogeneous Markov process and, hence, is a Markov chain. Letting $\{\partial\}$ be an absorbing state corresponding to $J(\infty)$, we note that $J\left(\tau_{x}\right)$ lives on $E_{+} \cup\{\partial\}$, because $X(t)$ cannot hit a new maximum when $J(t)$ is in a state corresponding to a downward subordinator; see also [16]. Let $\Lambda(q)$ be the $\left(N_{+} \times N_{+}\right)$-dimensional transition rate matrix of $J\left(\tau_{x}\right)$ restricted to $E_{+}$, that is,

$$
\mathrm{P}\left(J\left(\tau_{x}\right)=j, \tau_{x}<e_{q} \mid J\left(\tau_{0}\right)=i\right)=\left(\mathrm{e}^{\Lambda(q) x}\right)_{i j} \quad \text { for } i, j \in E_{+} .
$$


This, in fact, shows that the first passage process $\left(\tau_{x}, J\left(\tau_{x}\right)\right), x \geq 0$, is a MAP itself, and $\Lambda(-q)$ is its matrix exponent: $\mathrm{E}_{J\left(\tau_{0}\right)}\left[\mathrm{e}^{-q \tau_{x}} ; J\left(\tau_{x}\right)\right]=\mathrm{e}^{\Lambda(q) x}$.

Another matrix of interest is the $N \times N_{+}$matrix $\Pi(q)$ defined by

$$
\Pi(q)_{i j}=\mathrm{P}_{i}\left(J\left(\tau_{0}\right)=j, \tau_{0}<e_{q}\right) \quad \text { for } i \in E \text { and } j \in E_{+} .
$$

This matrix specifies initial distributions of the time-changed Markov chain $J\left(\tau_{x}\right)$, so that $\mathrm{E}\left[\mathrm{e}^{-q \tau_{x}} ; J\left(\tau_{x}\right)\right]=\Pi(q) \mathrm{e}^{\Lambda(q) x}$. Note also that $\Pi(q)$ restricted to the rows in $E_{+}$is the identity matrix, because $\tau_{0}=0$ a.s. when $J(0) \in E_{+}[15$, Theorem 6.5]. We note that the case in which $q=0$ is a special case corresponding to no killing. In order to simplify the notation, we often write $\Lambda$ and $\Pi$ instead of $\Lambda(0)$ and $\Pi(0)$.

It is noted that if $q>0$ or $q=0, \kappa<0$, then $\Lambda(q)$ is a transient transition rate matrix: $\Lambda(q) \mathbf{1}_{+} \leq \mathbf{0}_{+}$, with at least one strict inequality. If, however, $\kappa \geq 0$ then $\Lambda$ is a recurrent transition rate matrix: $\Lambda \mathbf{1}_{+}=\mathbf{0}_{+} ;$also, $\Pi \mathbf{1}_{+}=\mathbf{1}$. These statements follow trivially from $[2$, Proposition XI.2.10]. Finally, note that $\Lambda$ is an irreducible matrix, because so is $Q$. Hence, if $\Lambda$ is recurrent then, by the Perron-Frobenius theory [2, Theorem I.6.5], the eigenvalue 0 is simple, because it is the eigenvalue with maximal real part.

It is instructive to consider the 'degenerate' MAP, i.e. the MAP with dimension $N=1$. Such a MAP is just a Lévy process, and $\Lambda(q)=-\Phi(q)$, where $\Phi(q)$ is the right inverse of $\psi(\alpha), \alpha \geq 0$. Note also that $\Lambda$ being recurrent (and, hence, singular) corresponds to $\Phi(0)=0$.

\section{Preliminaries}

In this section we review some basic facts from analytic matrix function theory. Let $A(z)$ be an analytic matrix function $((n \times n)$-dimensional $)$, defined on some domain $D \subset \mathbb{C}$, where it is assumed that $\operatorname{det}(A(z))$ is not identically 0 on this domain. For any $\lambda \in D$, we can write

$$
A(z)=\sum_{i=0}^{\infty} \frac{1}{i !} A^{(i)}(\lambda)(z-\lambda)^{i},
$$

where $A^{(i)}(\lambda)$ denotes the $i$ th derivative of $A(z)$ at $\lambda$. We say that $\lambda$ is an eigenvalue of $A(z)$ if $\operatorname{det}(A(\lambda))=0$.

Definition 1. We say that the vectors $\boldsymbol{v}_{0}, \ldots, \boldsymbol{v}_{r-1} \in \mathbb{C}^{n}$ with $\boldsymbol{v}_{0} \neq \mathbf{0}$ form a Jordan chain of $A(z)$ corresponding to the eigenvalue $\lambda$ if

$$
\sum_{i=0}^{j} \frac{1}{i !} A^{(i)}(\lambda) \boldsymbol{v}_{j-i}=\mathbf{0}, \quad j=0, \ldots, r-1 .
$$

Note that this definition is a generalization of the well-known notion of a Jordan chain for a square matrix $A$. In this classical case $A(z)=z \mathbb{I}-A$, and (3) reduces to

$$
A \boldsymbol{v}_{0}=\lambda \boldsymbol{v}_{0}, \quad A \boldsymbol{v}_{1}=\lambda \boldsymbol{v}_{1}+\boldsymbol{v}_{0}, \ldots, \quad A \boldsymbol{v}_{r-1}=\lambda \boldsymbol{v}_{r-1}+\boldsymbol{v}_{r-2}
$$

The following result is well known [10] and is an immediate consequence of (4).

Proposition 1. Let $\boldsymbol{v}_{0}, \ldots, \boldsymbol{v}_{r-1}$ be a Jordan chain of $A(z)$ corresponding to the eigenvalue $\lambda$, and let $C(z)$ be $a(m \times n)$-dimensional matrix. If $B(z)=C(z) A(z)$ is $r-1$ times differentiable at $\lambda$ then

$$
\sum_{i=0}^{j} \frac{1}{i !} B^{(i)}(\lambda) v_{j-i}=\mathbf{0}, \quad j=0, \ldots, r-1 .
$$


Note that if $B(z)$ is a square matrix then $\boldsymbol{v}_{0}, \ldots, \boldsymbol{v}_{r-1}$ is a Jordan chain of $B(z)$ corresponding to the eigenvalue $\lambda$. It is, however, not required that $C(z)$ and $B(z)$ be square matrices.

Let $m$ be the multiplicity of $\lambda$ as a 0 of $\operatorname{det}(A(z))$, and let $p$ be the dimension of the null space of $A(\lambda)=A_{0}$. It is known (see, for example, [10]) that there exists a canonical system of Jordan chains corresponding to $\lambda$,

$$
\boldsymbol{v}_{0}^{(k)}, \boldsymbol{v}_{1}^{(k)}, \ldots, \boldsymbol{v}_{r_{k}-1}^{(k)}, \quad k=1, \ldots, p,
$$

such that the vectors $\boldsymbol{v}_{0}^{(1)}, \ldots, \boldsymbol{v}_{0}^{(p)}$ form the basis of the null space of $A_{0}$ and $\sum_{i=1}^{p} r_{i}=m$. We write such a canonical system of Jordan chains in matrix form:

$$
V=\left[\boldsymbol{v}_{0}^{(1)}, \boldsymbol{v}_{1}^{(1)}, \ldots, \boldsymbol{v}_{r_{1}-1}^{(1)}, \ldots, \boldsymbol{v}_{0}^{(p)}, \boldsymbol{v}_{1}^{(p)}, \ldots, \boldsymbol{v}_{r_{p}-1}^{(p)}\right], \quad \Gamma=\operatorname{diag}\left[\Gamma^{(1)}, \ldots, \Gamma^{(p)}\right],
$$

where $\Gamma^{(i)}$ is the Jordan block of size $r_{i} \times r_{i}$ with eigenvalue $\lambda$, i.e. a square matrix having $0 \mathrm{~s}$ everywhere except along the diagonal, whose elements are equal to $\lambda$, and the superdiagonal, whose elements are equal to 1 .

Definition 2. A pair of matrices $(V, \Gamma)$ given by (5) is called a Jordan pair of $A(z)$ corresponding to the eigenvalue $\lambda$.

We note that, unlike in the classical case, the vectors forming a Jordan chain are not necessarily linearly independent; furthermore, a Jordan chain may contain a null vector.

We conclude this section with a result on entire functions of matrices defined through

$$
f(M)=\sum_{i=0}^{\infty} \frac{1}{i !} f^{(i)}(0) M^{i}
$$

for an entire function $f: \mathbb{C} \rightarrow \mathbb{C}$ and a square matrix $M$. The next lemma will be important for applications and is immediate from [9, Theorem 6.6].

Lemma 1. Let $f: \mathbb{C} \rightarrow \mathbb{C}$ be an entire function, and let $\Gamma$ be a Jordan block of size $k$ with $\lambda$ on the diagonal. Then, for an arbitrary set of vectors $\boldsymbol{v}_{0}, \ldots, \boldsymbol{v}_{k-1}$, the $(j+1)$ th column of the matrix $\left[\boldsymbol{v}_{0}, \ldots, \boldsymbol{v}_{k-1}\right] f(\Gamma)$ equals

$$
\sum_{i=0}^{j} \frac{1}{i !} f^{(i)}(\lambda) v_{j-i}, \quad j=0, \ldots, k-1 .
$$

\section{Jordan normal form of $\Lambda(q)$}

In this section we consider a spectrally negative MAP $(X(t), J(t))$ with matrix exponent $F(\alpha)$ and asymptotic drift $\kappa$. Let $\lambda_{1}, \ldots, \lambda_{k}$ be the eigenvalues of $F^{q}(\alpha)$, to be understood as the 0 s of $\operatorname{det}\left(F^{q}(\alpha)\right)$ for a given $q \geq 0$, in its region of analyticity $\mathbb{C}^{\operatorname{Re}>0}$. Let $\left(V_{i}, \Gamma_{i}\right)$ be a Jordan pair corresponding to the eigenvalue $\lambda_{i}$. Define the matrices $V$ and $\Gamma$ in the following way:

$$
\begin{gathered}
V=\left[V_{1}, \ldots, V_{k}\right] \quad \text { and } \quad \Gamma=\operatorname{diag}\left[\Gamma_{1}, \ldots, \Gamma_{k}\right] \quad \text { if } q>0 \text { or } q=0, \kappa<0 \\
V=\left[\mathbf{1}, V_{1}, \ldots, V_{k}\right] \quad \text { and } \quad \Gamma=\operatorname{diag}\left[0, \Gamma_{1}, \ldots, \Gamma_{k}\right] \quad \text { if } q=0, \kappa \geq 0
\end{gathered}
$$

Let the matrices $V_{+}$and $V_{\downarrow}$ be the restrictions of the matrix $V$ to the rows corresponding to $E_{+}$ and $E_{\downarrow}$, respectively. 
Theorem 1. It holds that $\Gamma$ and $V_{+}$are $\left(N_{+} \times N_{+}\right)$-dimensional matrices, $V_{+}$is invertible, and

$$
\Lambda(q)=-V_{+} \Gamma V_{+}^{-1}, \quad \Pi(q)=V V_{+}^{-1} .
$$

This theorem extends the results of [1, Section 5], [6, Section 7], and [20, Section 8]. Importantly, it is no longer necessary to assume that the eigenvalues of $F^{q}(\alpha)$ are semi-simple, which is not always the case, as shown in [13, Example 2.1]. The generality of Theorem 1 allows us to prove a number of further identities, as alluded to and then demonstrated in Section 4.

We start by establishing a lemma, which can be considered as a weak analog of Theorem 1 .

Lemma 2. If $\boldsymbol{v}^{0}, \ldots, \boldsymbol{v}^{r-1}$ is a Jordan chain of $F^{q}(\alpha)$ corresponding to the eigenvalue $\lambda \in$ $\mathbb{C}^{\operatorname{Re}>0}$ then $\boldsymbol{v}_{+}^{0}, \ldots, \boldsymbol{v}_{+}^{r-1}$ is a Jordan chain of $\alpha \mathbb{I}+\Lambda(q)$ corresponding to the eigenvalue $\alpha=\lambda$ and $\Pi(q) \boldsymbol{v}_{+}^{i}=\boldsymbol{v}^{i}$ for $i=0, \ldots, r-1$.

Proof. It is known from [3, Theorem 2.1] that, for $\alpha \in \mathbb{C}^{\operatorname{Re}>0}$,

$$
M_{\alpha}(t)=\left[\int_{0}^{t} \mathrm{e}^{\alpha X(s)} \boldsymbol{e}_{J(s)}^{\top} \mathrm{d} s\right] \cdot F(\alpha)+\boldsymbol{e}_{k}^{\top}-\mathrm{e}^{\alpha X(t)} \boldsymbol{e}_{J(t)}^{\top},
$$

is a row vector valued local martingale under the probability measure $\mathrm{P}_{k}$. Furthermore, the stopped process $M_{\alpha}\left(t \wedge \tau_{x} \wedge e_{q}\right)$ is a martingale, which yields $\mathrm{E}_{k} M_{\alpha}\left(t \wedge \tau_{x} \wedge e_{q}\right)=\mathbf{0}^{\top}$. Note that

$$
\mathrm{E}_{k}\left[\mathrm{e}^{\alpha X\left(e_{q}\right)} 1_{\left\{t \wedge \tau_{x}>e_{q}\right\}} \boldsymbol{e}_{J\left(e_{q}\right)}^{\top}\right]=q \mathrm{E}_{k}\left[\int_{0}^{t \wedge \tau_{x} \wedge e_{q}} \mathrm{e}^{\alpha X(s)} \boldsymbol{e}_{J(s)}^{\top} \mathrm{d} s\right],
$$

to obtain

$$
C(\alpha) F^{q}(\alpha)=B(\alpha)
$$

where

$$
C(\alpha)=\mathrm{E}_{k}\left[\int_{0}^{t \wedge \tau_{x} \wedge e_{q}} \mathrm{e}^{\alpha X(s)} \boldsymbol{e}_{J(s)}^{\top} \mathrm{d} s\right], \quad B(\alpha)=\mathrm{E}_{k}\left[\mathrm{e}^{\alpha X\left(t \wedge \tau_{x}\right)} 1_{\left\{t \wedge \tau_{x}<e_{q}\right\}} \boldsymbol{e}_{J\left(t \wedge \tau_{x}\right)}^{\top}\right]-\boldsymbol{e}_{k}^{\top} .
$$

Noting that $X(\cdot) \leq x$ on $\left[0, \tau_{x}\right]$ and using the usual dominated convergence argument, we conclude that $B(\alpha)$ is infinitely differentiable in $\alpha \in \mathbb{C}^{\mathrm{Re}>0}$. Apply Proposition 1 to (7) to see that, for all $j=0, \ldots, r-1$, the following holds true:

$$
\sum_{i=0}^{j} \frac{1}{i !} \mathrm{E}_{k}\left[X^{i}\left(t \wedge \tau_{x}\right) \mathrm{e}^{\lambda X\left(t \wedge \tau_{x}\right)} 1_{\left\{t \wedge \tau_{x}<e_{q}\right\}} \boldsymbol{e}_{J\left(t \wedge \tau_{x}\right)}^{\top}\right] \boldsymbol{v}^{j-i}-\boldsymbol{e}_{k}^{\top} \boldsymbol{v}^{j}=0
$$

Letting $t \rightarrow \infty$ we obtain

$$
\sum_{i=0}^{j} \frac{1}{i !} x^{i} \mathrm{e}^{\lambda x} \mathrm{P}_{k}\left(J\left(\tau_{x}\right), \tau_{x}<e_{q}\right) \boldsymbol{v}^{j-i}-\boldsymbol{e}_{k}^{\top} \boldsymbol{v}^{j}=0
$$

where $\mathrm{P}_{k}\left(J\left(\tau_{x}\right), \tau_{x}<e_{q}\right)$ denotes a row vector with $\ell$ th element given by $\mathrm{P}_{k}\left(J\left(\tau_{x}\right)=\ell\right.$, $\left.\tau_{x}<e_{q}\right)$. Note that the case when $q=0$ and $\mathrm{P}_{k}\left(\tau_{x}=\infty\right)>0$ should be treated with care. In this case $\kappa<0$ and, thus, $\lim _{t \rightarrow \infty} X(t)=-\infty$ a.s. [2, Proposition XI.2.10], so the above limit is still valid. 
Considering (10) for all $k \in E$ and choosing $x=0$, we indeed obtain $\Pi(q) \boldsymbol{v}_{+}^{j}=\boldsymbol{v}^{j}$. If, however, we pick $k \in E_{+}$then

$$
\sum_{i=0}^{j} \frac{1}{i !} x^{i} \mathrm{e}^{(\lambda I+\Lambda(q)) x} \boldsymbol{v}_{+}^{j-i}-\boldsymbol{v}_{+}^{j}=\mathbf{0}_{+} .
$$

Take the right derivative in $x$ at 0 of both sides to see that

$$
(\lambda I+\Lambda(q)) \boldsymbol{v}_{+}^{j}+\boldsymbol{v}_{+}^{j-1}=\mathbf{0}_{+},
$$

which shows that $\boldsymbol{v}_{+}^{0}, \ldots, \boldsymbol{v}_{+}^{r-1}$ is a Jordan chain of $\alpha \mathbb{I}+\Lambda(q)$ corresponding to the eigenvalue $\lambda$.

We are now ready to give a proof of our main result, Theorem 1.

Proof of Theorem 1. Lemma 2 states that $\boldsymbol{v}_{+}^{0}, \ldots, \boldsymbol{v}_{+}^{r-1}$ is a classical Jordan chain of the matrix $-\Lambda(q)$. Recall that if $q=0$ and $\kappa \geq 0$, then $\Lambda(q) \mathbf{1}_{+}=\mathbf{0}_{+}$and $\Pi(q) \mathbf{1}_{+}=\mathbf{1}$. Therefore, the columns of $V_{+}$are linearly independent [11, Proposition 1.3.4] and

$$
-\Lambda(q) V_{+}=V_{+} \Gamma, \quad \Pi(q) V_{+}=V .
$$

Consider the case when $q>0$. Now [13, Theorem 1] states that $\operatorname{det}\left(F^{q}(\alpha)\right)$ has $N_{+}$ zeros (counting multiplicities) in $\mathbb{C}^{\operatorname{Re}>0}$, so the matrices $V_{+}$and $\Gamma$ are of size $N_{+} \times N_{+}$by construction (6). Note that there is a one-to-one correspondence between the $0 \mathrm{~s}$ of $\operatorname{det}\left(F^{q}(\alpha)\right)$ in $\mathbb{C}^{\mathrm{Re}>0}$ and the eigenvalues of $-\Lambda(q)$ when $q>0$.

Assume now that $q=0$. We need to only show that $\operatorname{det}(F(\alpha))$ has $N_{+}-1_{\{\kappa \geq 0\}}$ zeros (counting multiplicities) in $\mathbb{C}^{\mathrm{Re}>0}$. Pick a sequence of $q_{n}$ converging to 0 and consider a sequence of matrix exponents $F^{q_{n}}(\alpha)=F(\alpha)-q_{n} \mathbb{I}$ and transition rate matrices $\Lambda\left(q_{n}\right)$. From (2), it follows that $\mathrm{e}^{\Lambda\left(q_{n}\right)} \rightarrow \mathrm{e}^{\Lambda}$; hence, the eigenvalues of $\Lambda\left(q_{n}\right)$ converge to the eigenvalues of $\Lambda$ (preserving multiplicities) as $n \rightarrow \infty$. Moreover, all the eigenvalues of $\Lambda$ have negative real part except a simple one at 0 if $\kappa \geq 0$. The abovementioned one-to-one correspondence and the convergence statement of [13, Theorem 9] complete the proof.

The above proof strengthens [13, Theorem 2]; we remove the assumption that $\kappa$ is nonzero and finite.

Corollary 1. It holds that $\operatorname{det}(F(\alpha))$ has $N_{+}-1_{\{\kappa \geq 0\}}$ zeros (counting multiplicities) in $\mathbb{C}^{\mathrm{Re}>0}$.

\section{Applications}

A number of applications of our result is discussed in detail in the extended version of this paper [7]. These applications include finding the stationary distributions of a one-sided MAP reflected at 0 , and a Markov-modulated Brownian motion (MMBM) reflected to stay in the strip $[0, B]$. Moreover, we solve a two-sided exit problem for an MMBM. It is noted that an MMBM is a MAP with continuous paths and, hence, our result can be applied to both $X(t)$ and $-X(t)$, which hints why the two-sided problems become tractable.

The approach to the above problems consists of the following steps:

- use a martingale argument to arrive at an initial equation involving the unknown quantities and $F(\alpha)$,

- use the properties of Jordan chains, such as Proposition 1 and Lemma 1, to rewrite the initial equation in terms of $(V, \Gamma)$, 
- use the special structure of $(V, \Gamma)$, such as the invertibility of $V$, to simplify the equation,

- eliminate the Jordan pair by introducing $\Lambda$ and $\Pi$ using Theorem 1 to recover the probabilistic interpretation of the involved matrices and claim uniqueness of the solution.

It is noted that this approach can be seen as an extension of the ideas known as 'martingale calculations for MAPs' [2, Chapter XI, 4a] to its final and general form. It is important that no assumptions about the number and simplicity of the eigenvalues are needed. For some problems, certain eigenvalues are inherently nonsimple. For example, the special but important case of an MMBM with zero drift immediately leads to a nonsimple eigenvalue 0 . In this case an additional equation associated to the null Jordan chain is required to obtain the solution; see also [21]. In our framework, this equation comes out in a natural way.

\subsection{Matrix integral equation}

In this section we demonstrate how our result can be used to show in a simple way that a pair $(\Pi(q), \Lambda(q))$ is a unique solution of a certain matrix integral equation. This equation under different assumptions appears in [1], [6], [8], [17], [18], [20], and [21].

Define two sets of matrices: let $\mathcal{M}$ be a set of all $N_{+} \times N_{+}$irreducible transition rate matrices and let $\mathcal{P}$ be a set of $N \times N_{+}$matrices $P$ satisfying $P_{+}=\mathbb{I}$. Furthermore, partition $\mathcal{M}$ into two disjoint sets, $\mathcal{M}_{0}$ and $\mathcal{M}_{1}$, of transient and recurrent matrices, respectively. Clearly, $\Lambda(q) \in \mathcal{M}_{i}, i=1_{\{q=0, \kappa \geq 0\}}$, and $\Pi(q) \in \mathcal{P}$. We use the following notation for arbitrary matrices $P \in \mathcal{P}$ and $-M \in \mathcal{M}$ :

$$
\begin{aligned}
F^{q}(P, M)= & \Delta_{\boldsymbol{a}} P M+\frac{1}{2} \Delta_{\boldsymbol{\sigma}}^{2} P M^{2}+\int_{-\infty}^{0} \Delta_{\boldsymbol{v}}(\mathrm{d} x) P\left(\mathrm{e}^{M x}-\mathbb{I}-M x 1_{\{x>-1\}}\right) \\
& +\int_{-\infty}^{0} Q \circ G(\mathrm{~d} x) P \mathrm{e}^{M x}-q P
\end{aligned}
$$

where $\left(a_{i}, \sigma_{i}, v_{i}(\mathrm{~d} x)\right)$ are the Lévy triplets corresponding to the Lévy processes $X_{i}(\cdot)$, that is, $\psi_{i}(\alpha)=a_{i} \alpha+\sigma_{i}^{2} / 2 \alpha^{2}+\int_{-\infty}^{0}\left(\mathrm{e}^{\alpha x}-1-\alpha x 1_{\{x>-1\}}\right) \nu_{i}(\mathrm{~d} x)$, and $G_{i j}(\mathrm{~d} x)$ is the distribution of $U_{i j}$. It will be clear from the following that the integrals converge for the above choices of $P$ and $M$.

We use (1) to denote the matrix of zeros of appropriate dimension.

Theorem 2. It holds that $(\Pi(q), \Lambda(q))$ is the unique pair $(P, M) \in \mathcal{P} \times \mathcal{M}_{i}$ with $i=$ $1_{\{q=0, \kappa \geq 0\}}$ which satisfies $F^{q}(P,-M)=\mathbb{O}_{+}$.

Proof. In the proof we drop the superscript $q$ to simplify notation. Let $-M=V_{+} \Gamma V_{+}^{-1}$ be a Jordan decomposition of the matrix $-M$. We can extend $V_{+}$to a $N \times N_{+}$matrix $V$ through $V=P V_{+}$, because $P_{+}=\mathbb{I}$. Also, let $\boldsymbol{v}_{+}^{0}, \ldots, \boldsymbol{v}_{+}^{r-1}$ be the columns of $V$ corresponding to some Jordan block of size $r$ and eigenvalue $\lambda$. Observe that $\lambda \in \mathbb{C}^{\operatorname{Re}>0}$ or $\lambda=0$, in which case it must be simple, because $M \in \mathcal{M}$. Note that $g(-M)=V_{+} g(\Gamma) V_{+}^{-1}$ for an entire function $g: \mathbb{C} \rightarrow \mathbb{C}$, and use Lemma 1 to see that the column of $F(P,-M) V_{+}$corresponding to $\boldsymbol{v}_{+}^{j}, j=0, \ldots, r-1$, equals

$$
\sum_{i=0}^{j} \frac{1}{i !} F^{(i)}(\lambda) P \boldsymbol{v}_{+}^{j-i}=\sum_{i=0}^{j} \frac{1}{i !} F^{(i)}(\lambda) \boldsymbol{v}^{j-i} .
$$

We also used the fact that differentiation of $F(\alpha)$ at $\lambda, \operatorname{Re}(\lambda)>0$, can be done under the integral signs and no differentiation is needed for a simple eigenvalue $\lambda=0$, if such exists. 
If $M=\Lambda$ and $P=\Pi$, then according to Theorem 1 the matrices $V$ and $\Gamma$ can be chosen as in (6). Hence, (11) becomes $\mathbf{0}_{+}$, because $\boldsymbol{v}^{0}, \ldots, \boldsymbol{v}^{r-1}$ is a Jordan chain of $F(\alpha)$; see (3). But $V_{+}$is an invertible matrix and so $F(\Pi,-\Lambda)=\mathbb{O}_{+}$.

Suppose now that $F(P,-M)=\mathbb{O}_{+}$with $M \in \mathcal{M}_{i}$ and $P \in \mathcal{P}$. Then the vectors $\boldsymbol{v}^{0}, \ldots, \boldsymbol{v}^{r-1}$ form a Jordan chain of $F(\alpha)$ corresponding to an eigenvalue $\lambda \in \mathbb{C}^{\operatorname{Re}>0}$ or $\lambda=0$. If $q=0, \kappa \geq 0$, and $\lambda=0$, which is a simple eigenvalue of $M$, then $F(0) \boldsymbol{v}^{0}=Q \boldsymbol{v}^{0}=\mathbf{0}$ implies that $\boldsymbol{v}^{0}=c \mathbf{1}$, where $c \neq 0$ is a constant. Combining this observation and Lemma 2 we obtain $\Lambda V_{+}=-V_{+} \Gamma$ and $\Pi V_{+}=V$, and, hence, $M=\Lambda$ and $P=\Pi$.

Remark 1. It is easy to see from the above proof that the sets $\mathcal{M}_{0}$ and $\mathcal{M}_{1}$ of respectively transient and recurrent irreducible transition matrices can be enlarged so that Theorem 2 still holds. Namely, we can enlarge $\mathcal{M}_{0}$ to include all $N_{+} \times N_{+}$matrices with eigenvalues in $\mathbb{C}^{\mathrm{Re}<0} ; M_{1}$ is taken to be a set of all $N_{+} \times N_{+}$matrices with eigenvalues in $\mathbb{C}^{\mathrm{Re}<0}$ and a simple eigenvalue at 0 .

From the above theorem we immediately obtain the following corollaries.

Corollary 2. If $N=N_{+}$then $M=\Lambda(q)$ is the unique solution of $F^{q}(\mathbb{I},-M)=\mathbb{O}$, where $M \in \mathcal{M}_{i}, i=1_{\{q=0, \kappa \geq 0\}}$.

For the case of an MMBM, i.e. a continuous MAP, we obtain a generalization of the results in [1] and [21].

Corollary 3. If $(X(t), J(t))$ is an MMBM then $(\Pi(q), \Lambda(q))$ is the unique pair $(P, M) \in$ $\mathcal{P} \times \mathcal{M}_{i}$ with $i=1_{\{q=0, \kappa \geq 0\}}$ which satisfies

$$
\frac{1}{2} \Delta_{\sigma}^{2} P M^{2}-\Delta_{a} P M+(Q-q \mathbb{I}) P=\mathbb{O}_{+} .
$$

\section{Acknowledgements}

The first author was partially supported by the Spanish Ministry of Education and Science, under grants MTM2007-63140 and SEJ2007-64500, and by the Community of Madrid, under grants CCG08-UC3M/ESP-4162 and CCG07-UC3M/ESP-3389. Part of his research was done when he was visiting the Hebrew University of Jerusalem by partial support of Madrid University Carlos III Grant for Young Researchers' Mobility. The third author was partially supported by grant 964/06 from the Israel Science Foundation and the Vigevani Chair in Statistics.

\section{References}

[1] Asmussen, S. (1995). Stationary distributions for fluid flow models with or without Brownian noise, Commun. Statist. Stoch. Models 11, 21-49.

[2] Asmussen, S. (2003). Applied Probability and Queues (Appl. Math. 51), 2nd edn. Springer, New York.

[3] Asmussen, S. and Kella, O. (2000). A multi-dimensional martingale for Markov additive processes and its applications. Adv. Appl. Prob. 32, 376-393.

[4] Asmussen, S. And Pihlsgånd, M. (2007). Loss rates for Lévy processes with two reflecting barriers. Math. Operat. Res. 32, 308-321.

[5] Asmussen, S., Avram, F. and Pistorius, M. R. (2004). Russian and American put options under exponential phase-type Lévy models. Stoch. Process. Appl. 109, 79-111.

[6] Breuer, L. (2008). First passage times for Markov additive processes with positive jumps of phase type. J. Appl. Prob. 45, 779-799.

[7] D’Auria, B., Ivanovs, J., Kella, O. and Mandjes, M. (2010). First passage process of a Markov additive process, with applications to reflection problems. Preprint. Available at http;//arxiv.org/abs/1006.2965v1. 
[8] Dieker, A. B. And Mandjes, M. (2010). Extremes of Markov-additive processes with one-sided jumps, with queueing applications. To appear in Methodology Comput. Appl. Prob. Available at http://www2.isye.gatech.edu/ adieker3/publications/modulatedfluid.pdf.

[9] DoolitTle, E. (1998). Analytic Functions of Matrices Available at http://citeseerx.ksu.edu.sa/viewdoc/ download?doi=10.1.1.51.2968\&rep=rep1\&type $=$ pdf.

[10] Gohberg, I. and Rodman, L. (1981). Analytic matrix functions with prescribed local data. J. Analyse Math. 40, 90-128.

[11] Gohberg, I., Lancaster, P. and Rodman, L. (2006). Invariant Subspaces of Matrices with Applications (Classics Appl. Math. 51). Society for Industrial and Applied Mathematics, Philadelphia, PA.

[12] Ivanovs, J. And Mandjes, M. (2010). First passage of time-reversible spectrally negative Markov additive processes. Operat. Res. Lett. 38, 77-81.

[13] Ivanovs, J., Boxma, O. And Mandjes, M. (2010). Singularities of the matrix exponent of a Markov additive process with one-sided jumps. Stoch. Process. Appl. 120, 1776-1794.

[14] Jobert, A. And Rogers, L. C. G. (2006). Option pricing with Markov-modulated dynamics. SIAM J. Control Optimization 44, 2063-2078.

[15] Kyprianou, A. E. (2006). Introductory Lectures on Fluctuations of Lévy Processes with Applications. Springer, Berlin.

[16] Kyprianou, A. E. And PAlmowski, Z. (2008). Fluctuations of spectrally negative Markov additive processes. In Séminaire de Probabilités XLI (Lecture Notes Math. 1934), Springer, Berlin, pp. 121-135.

[17] Miyazawa, M. and Takada, H. (2002). A matrix exponential form for hitting probabilities and its application to a Markov-modulated fluid queue with downward jumps. J. Appl. Prob. 39, 604-618.

[18] Pistorius, M. (2006). On maxima and ladder processes for a dense class of Lévy processes. J. Appl. Prob. 43, 208-220.

[19] Prabhu, N. U. (1998). Stochastic Storage Processes (Appl. Math. 15), 2nd edn. Springer, New York.

[20] Prabhu, N. U. and Zhu, Y. (1989). Markov-modulated queueing systems. Queueing Systems 5, $215-245$.

[21] Rogers, L. C. G. (1994). Fluid models in queueing theory and Wiener-Hopf factorization of Markov chains. Ann. Appl. Prob. 4, 390-413. 